\title{
Towards the spatialization of ALPAO dms
}

\section{Laslandes}

M. Laslandes, "Towards the spatialization of ALPAO dms," Proc. SPIE 11852, International Conference on Space Optics - ICSO 2020, 118524J (11 June 2021); doi: 10.1117/12.2599663

SPIE Event: International Conference on Space Optics - ICSO 2021, 2021, Online SPIE. Only 


\section{International Conference on Space Optics-ICSO 2020}

Virtual Conference

30 March-2 April 2021

Edited by Bruno Cugny, Zoran Sodnik, and Nikos Karafolas
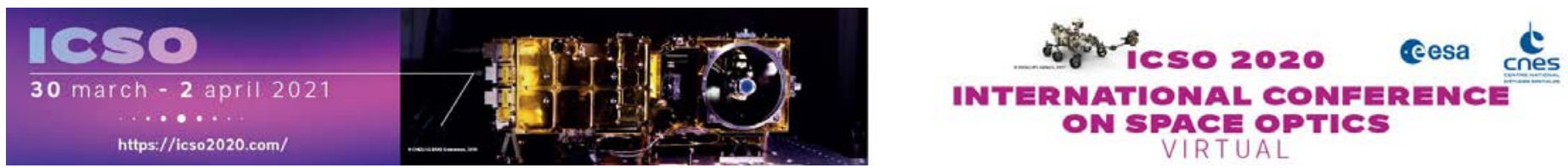

\section{Towards the spatialization of ALPAO dms}

\section{Cesa isopmeatians ecnes}




\title{
Towards the spatialization of ALPAO DMs
}

\author{
M. Laslandes ${ }^{1 \mathrm{a}}$ \\ ${ }^{a} A L P A O, 727$ rue Aristide Berges, 38330 Montbonnot Saint Martin, France
}

\begin{abstract}
ALPAO designs and manufactures a wide range of deformable mirrors (DM) for various applications, from large telescopes through laser or microelectronics to vision science and microscopy. In this paper we will present the recent developments towards a space use of our DMs. Thanks to the wide span of characteristics of ALPAO DMs, many applications in space telescopes can be considered: correction of surface error of a large primary mirror, correction of high spatial frequency errors for high contrast imaging, simplification of instrument design with an active surface, etc.

We present the ALPAO DM technology and the performances of DMs with large number of actuators. A focus will be made on the space application with the analysis of some correction cases and of the system reliability. The optimization of the DM's characteristics is presented, as well as the roadmap towards space qualification.
\end{abstract}

Keywords: Deformable Mirror, Space Telescope, Performance Optimization

\section{INTRODUCTION}

ALPAO designs and manufactures a wide range of deformable mirrors (DM) for various applications, from large telescopes through laser or microelectronics to vision science and microscopy.

Adaptive optics allows controlling the wave-front in telescopes, leading to more efficient instruments. This technique, widely used in Earth based astronomy [1], is now under study for space telescopes [2, 3]. Indeed, in this field, constraints on weight and compactness bring up the problem of structure stability. Future space observatories will have large lightweight primary mirrors, which will lose their best shapes, notably due to thermal variations and to gravity difference between integration on Earth and operations in space. Thus, it becomes mandatory to compensate for the induced aberrations with deformable mirrors. Moreover, polishing of large lightweight mirrors takes time and is not a trivial problem. The ability to compensate for the primary mirror surface error might reduce the specification on the mirror surface quality.

In addition to the correction of surface errors of the primary mirror, the presence of deformable mirrors in space telescopes might have other applications. For instance, the correction of high spatial frequency errors is essential for high contrast imaging. And a deformable surface in the optical train of the telescope could enable innovative optical designs, leading to simpler systems.

Thanks to the wide span of characteristics of ALPAO DMs, they are well suited for the applications mentioned above. In the first part of the paper, we present the ALPAO DM technology and the performances of DMs with large number of actuators. In the second part, we focus on the space application by analyzing some representative correction cases (which were provided by Airbus Defense and Space), and the reliability of the system. In the third part, we present the possibility to optimize the DM design and study the evolution of the performances depending on the DM characteristics. Finally, we talk about the perspectives for space qualification.

${ }^{1}$ marie.laslandes@alpao.fr; phone +33 476890 965; alpao.com 


\section{ALPAO DM TECHNOLOGY}

ALPAO DM technology is based on electro-magnetic actuation. The concept is presented in Figure 1: a continuous reflective surface is motioned by magnetic actuators. An actuator consists of an assembly of a coil, a magnet and a spring. By applying a current in the coils, the magnets are moved up and down to deform the membrane.

With such a technology, a large range of DM's characteristics can be achieved, with state of the arts performance.

The geometric characteristics of the DM can be chosen between the following ranges:

- number of actuators between 8 and 3228;

- $\quad$ pupil size between 7 and $240 \mathrm{~mm}$;

- $\quad$ actuator pitch between 0.8 and $20 \mathrm{~mm}$.

The resulting main DM performances are the following:

- resonance frequency between 200 and $3000 \mathrm{~Hz}$;

- $\quad$ stroke between 10 and $120 \mu \mathrm{m}$ PV;

- active flat as low as $3 \mathrm{~nm}$ RMS;

- $\quad$ less than $2 \mathrm{~nm}$ RMS evolution in 20 minutes, with the high stability option.

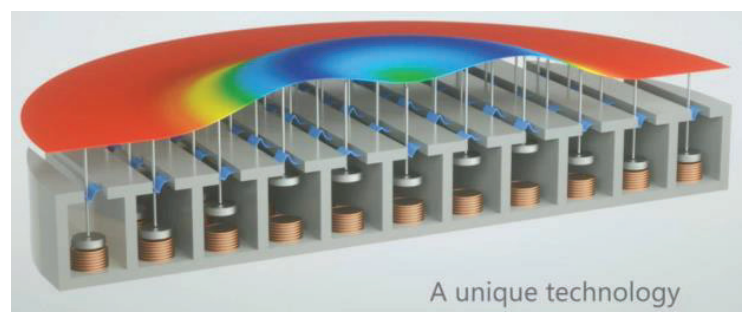

Figure 1: Concept of ALPAO Deformable Mirrors

We focus here on large number of actuators DM. Over the past years, ALPAO has developed 3 off-the-shelf large DMs (see

Table 1 and Figure 2). They are all organized in a regular square grid with a pitch of $1.5 \mathrm{~mm}$. As they were developed for applications in Earth-based astronomy [4], their design is prioritizing the speed over the stroke but we will see in Section 4 that the ratio speed/stroke can easily be adapted.

Figure 3 illustrates the high correction performance of such DMs: the best flat surface state of all DMs is lower than 7 nm RMS. It is realized in closed loop control by minimizing wavefront variations measured by a Shack-Hartmann wavefront sensor with $128 \times 128$ micro-lenses.

For the most demanding open-loop applications, high stability actuators are available on request. Figure 4 present a stability measurement performed on a DM468 with such option: the wavefront variation is less than $2 \mathrm{~nm}$ RMS over 20 minutes.

Table 1: ALPAO DM with a large number of actuators

\begin{tabular}{|c|c|c|c|}
\hline DM name & $\begin{array}{c}\text { Number of } \\
\text { actuator }\end{array}$ & $\begin{array}{c}\text { Number of actuators } \\
\text { across a diameter }\end{array}$ & $\begin{array}{c}\text { Pupil diameter } \\
(\mathbf{m m})\end{array}$ \\
\hline DM468 & 468 & 24 & 33 \\
\hline DM820 & 820 & 32 & 45 \\
\hline
\end{tabular}




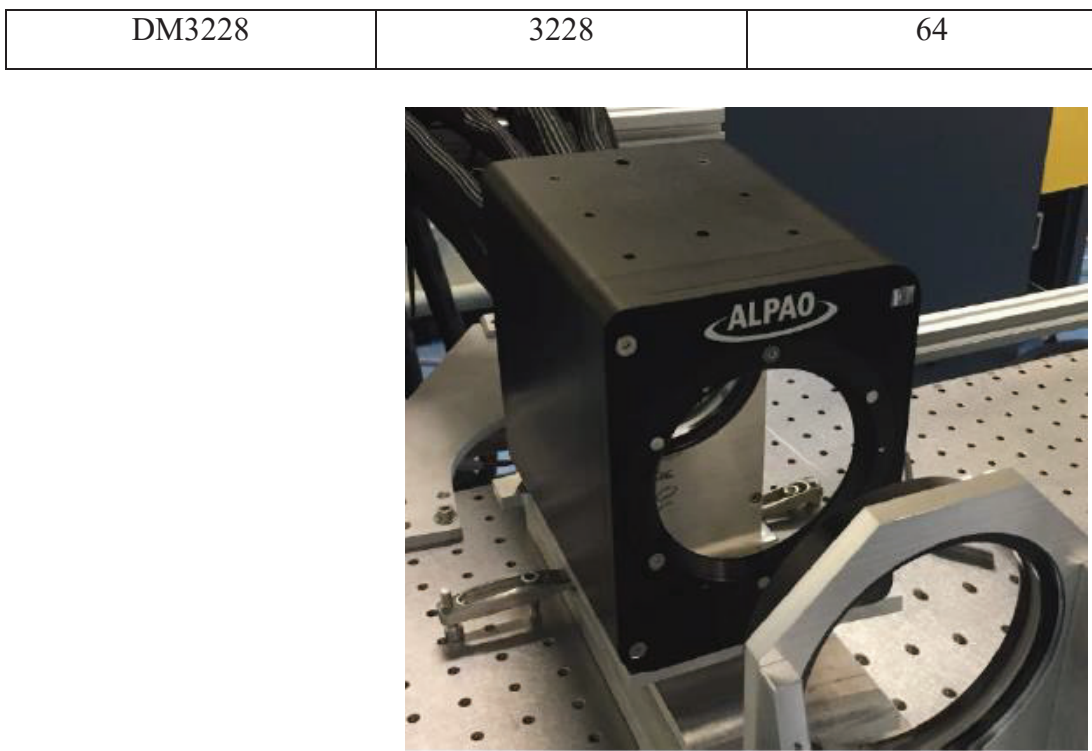

Figure 2: DM3228 installed at Observatoire Paris Meudon [5]

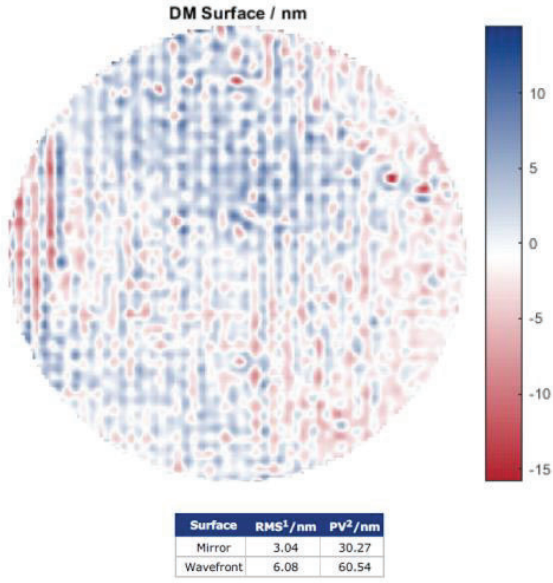

Figure 3: Best flat of a DM820, obtained in closed loop

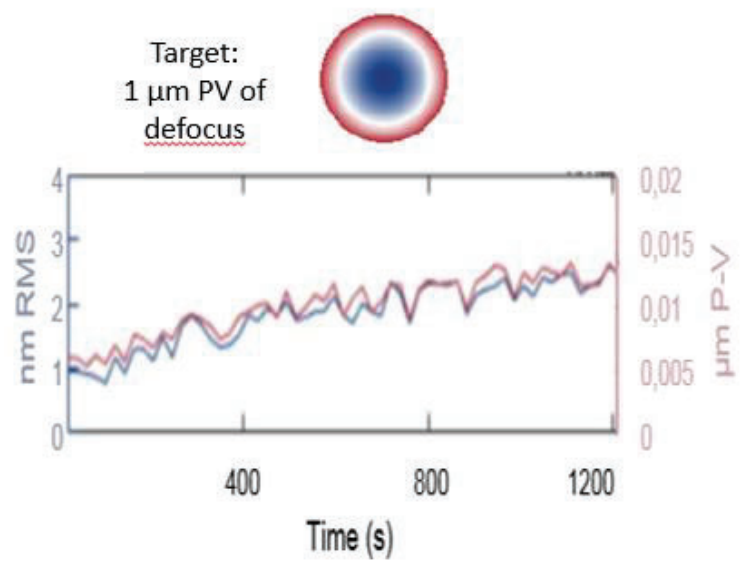

Figure 4: Stability of a focus shape obtained with a high stability DM468

\section{SPACE USE}

Given the span of characteristics, ALPAO DM are well suited for most of the space applications. As we will see in next Section, the DM's design (actuators pattern and characteristics, geometry, etc) can be adapted depending on the correction need. Thus, many cases can be considered, such as the correction of the surface errors of a large lightweight primary mirror, the correction of high spatial frequency errors for high contrast imaging, or the use of an active surface for instrument design simplification. In all this cases, the presence of a DM will improve the telescope performance, while relaxing other specifications. 
ALPAO DMs are currently at TRL4 and a roadmap for space qualification is defined. The main specific constraints to take into account are the weight, the compactness, the mechanical strength, the electronical and mechanical compliances, the power consumption and dissipation, the robustness and the reliability.

In this part, we focus on the correction performance of the current ALPAO DMs and on their reliability.

The performance of a DM3228 to correct for the expected error of a 4m class monolithic lightweight primary mirror are presented in Figure 5. The initial surface error map is created from a PSD simulating the expected error after mirror polishing and integration into the telescope. The influence functions of the DM3228 are used to compute the precision of correction of the phase map, and to predict the required actuators' commands. We can see that $91 \%$ of the initial error is corrected, and that the spatial frequencies below 10 cycle per pupil are efficiently suppressed. The residual surface map is $26 \mathrm{~nm}$ RMS, but the pattern is composed of really high spatial frequencies which can come from computation, sampling and interpolation noise. In this case, less than $20 \%$ of the actuator stroke is required to perform the correction. This example gives an idea of the expected performance for a DM3228. The residual error is mainly driven by the number of actuators so the correction precision is representative of any DM dimensioning (unless actuator saturation is reached). However the DM characteristics and the control law may impact the required stroke, and thus the power consumption.
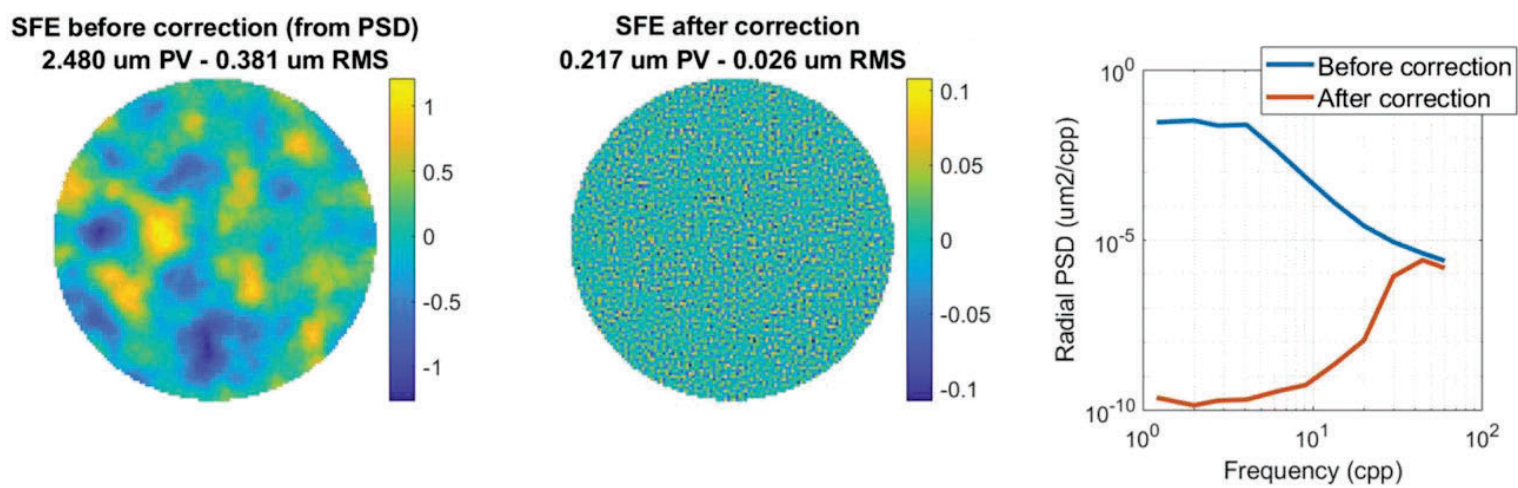

Figure 5: Correction of an expected shape error of a $4 \mathrm{~m}$ class primary mirror with a DM3228.

The input PSD was provided by Airbus Defense and Space.

Another interesting feature of ALPAO DMs is the low impact of non-functioning actuators. In this case, the actuator will not block the membrane into a fixed position but it will be floating, and thus it will follow the deformation induced by its neighbor actuator. This behavior is really advantageous and enables a good reliability of the system.

In order to characterize the impact of floating actuators, we study the correction of $1 \mu \mathrm{m}$ RMS of focus with a DM3228. In the simulation, we are simply forcing the commands of the non-functioning actuators to 0 . The performance of correction is studied with and without recalibration. Without recalibration means that the control matrix computed with all actuators is used. With recalibration means that a new control matrix is recomputed, without the floating actuators. Different actuators connection cases are modeled: the actuators can be organized in strips $(\sim 50$ actuators/strip), in clusters (of $4 \times 4$ or $8 \times 8$ ), or randomly (scrambling). The correction performance is computed for different number of floating actuators. As it will depend on the floating actuators position, 25 different configurations are randomly drawn and analyzed.

Figure 6 shows the impact of 128 floating actuators on the residual error after focus correction. For a fully functional system, the residual error amplitude is $6 \mathrm{~nm}$ RMS. Without recalibration, the floating actuators are clearly visible and the residual error is significantly impacted. On the other hand, when a recalibration is performed, the floating actuators have a really low impact on the performance. Figure 7 summarizes the performance evolution with different number of floating actuators. With recalibration, the residual error increases slowly and linearly with number of floating actuators. 
The actuators configuration that has the less impact is the random organization (which is equivalent to a scrambling in the DM cabling), then the strips.

The same study was done for other error shapes and for DMs with different number of actuators and led to the same conclusion. It is interesting to note that the correction of high spatial frequency errors seems to be less impacted by the loss of actuators.

In conclusion, we have shown that if there is a possibility to recalibrate the system, the impact of floating actuators is low. We can consider that it is acceptable for the DM performance to have up to $3 \%$ of floating actuators.
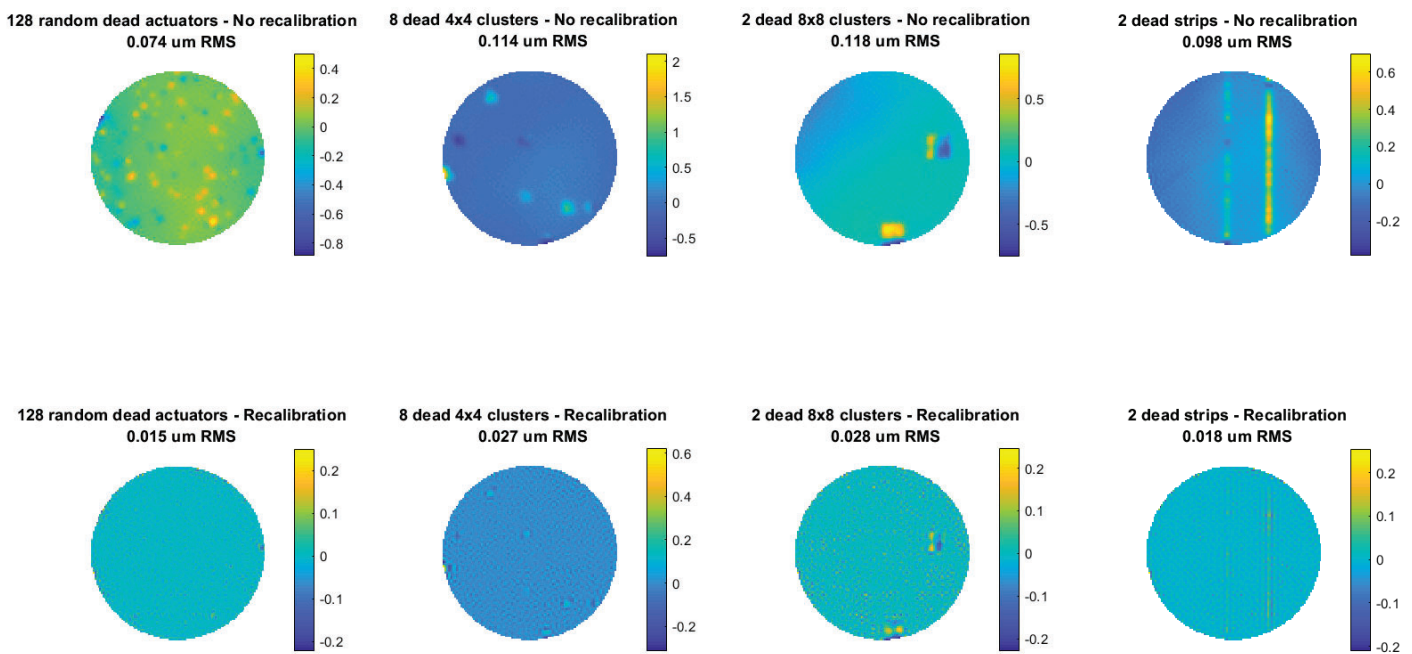

Figure 6: Correction of $1 \mu \mathrm{m}$ RMS of focus with 128 non-functioning actuators.

The residual error with a fully functional system is $6 \mathrm{~nm}$ RMS 


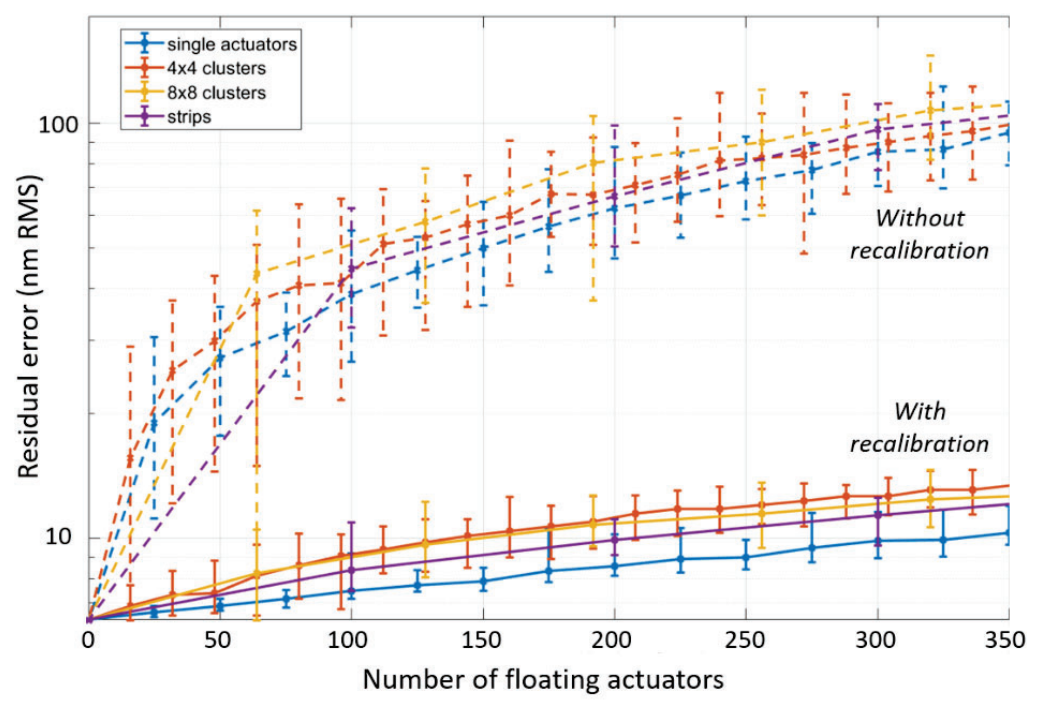

Figure 7: Fitting errors for the correction of $1 \mu \mathrm{m}$ RMS of focus with DM3228, for different configurations of floating actuators

\section{PERFORMANCE OPTIMIZATION}

There are many design variables for the DMs. The main ones are the actuator layout (number of actuators, pitch, and geometry of the pattern), the actuators stiffness and the mirror membrane geometry (diameter and thickness). All these parameters can be optimized depending on the correction needs. The generally considered outputs are the precision of correction, the speed of the correction loop and the stroke of the DM (maximum amplitude that can be corrected).

This Section presents some design considerations in order to choose an adequate design.

The number of actuators is driven by the spatial frequencies to be corrected. In order to illustrate this point, we adopted a simplified approach by modeling a square DM and studying the correction of a noise having a $1 / \mathrm{f}^{2}$ power spectrum. As an example, Figure 8 presents the correction performance of a DM with 36x36 actuators. The difference between the PSD of the wave-front before and after correction is giving the correction capabilities of the studied DM for each individual spatial frequency. Figure 9 is showing this result for different number of actuators. This is clearly illustrating the spatial frequency of actuators can be directly linked to the correction performance. Indeed, if $\mathrm{N}_{\mathrm{a}}$ is the number of actuators across a diameter of the DM:

- The spatial frequencies $<\mathrm{N}_{\mathrm{a}} / 3$ are almost entirely corrected

- The spatial frequencies $<\mathrm{N}_{\mathrm{a}} / 2$ are corrected up to $50 \%$

- The spatial frequencies $>\mathrm{N}_{\mathrm{a}}$ are not corrected

This is allowing to choose the right number of actuators. It is important to note here that there is a trade-off to do between precision of correction and power consumption. Indeed, the more actuators, the more power will be required. 


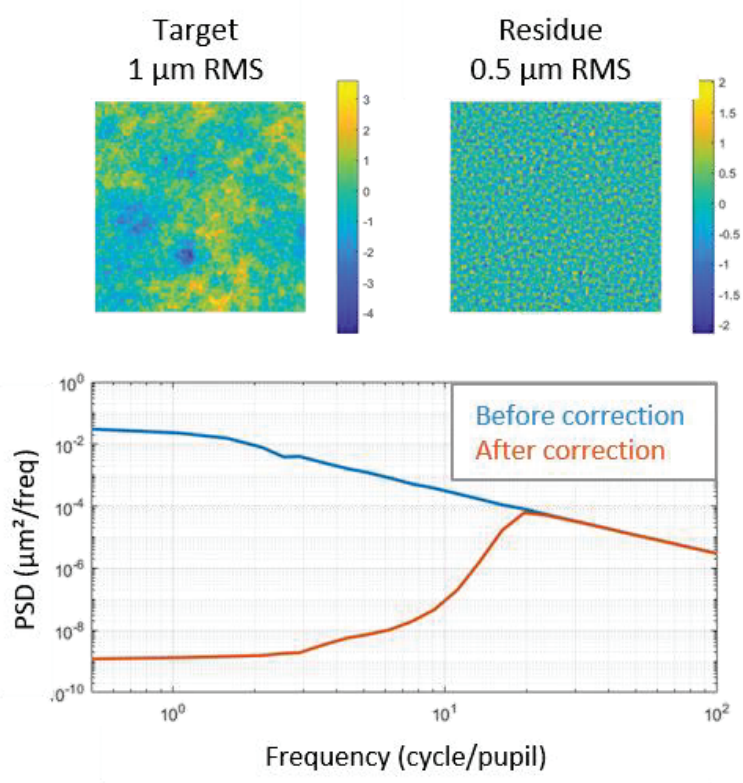

Figure 8: Example of correction for a DM36x36

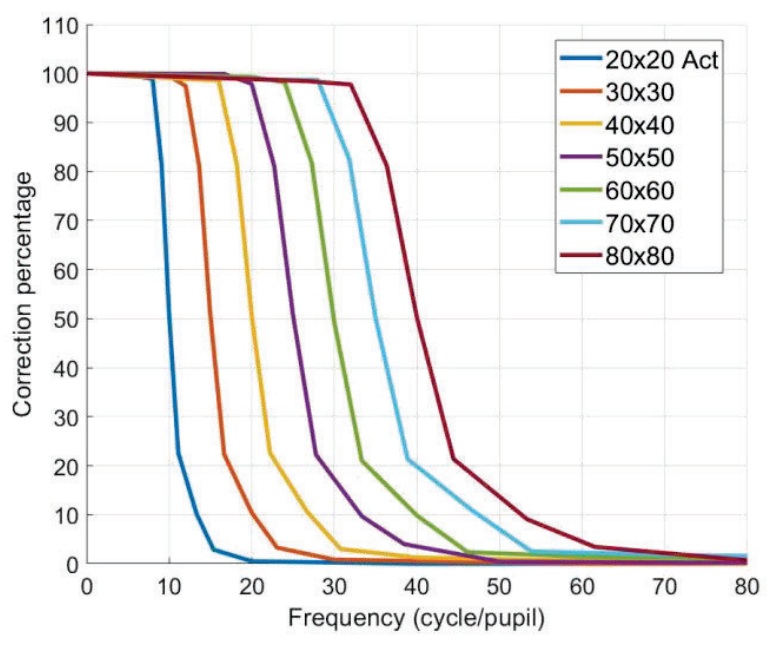

Figure 9: Correction performance for different DM configurations

Once the actuators' configuration is set, the main characteristics of the DM need to be chosen. The mirror thickness and the actuator stiffness will drive the performance of the DM, in terms of stroke and frequency (see Figure 10). We can note here that the electronics of the DM will also impact the stroke (and thus the power consumption and dissipation). We can see that the resonant frequency of the DM is mainly driven by the actuator stiffness, the inter-actuator stroke by the membrane thickness, and the $3 \times 3$ stroke by both characteristics.

As a first approximation, we can consider that the $3 \times 3$ stroke is representative of the low spatial frequencies stroke (such as tip, tilt and focus), and the inter-actuator stroke is representative of the high spatial frequencies stroke. In order to choose the right operating point of the DM, a trade-off needs to be made between speed and stroke. Moreover, the stroke choice will impact power consumption and actuator displacement resolution. 


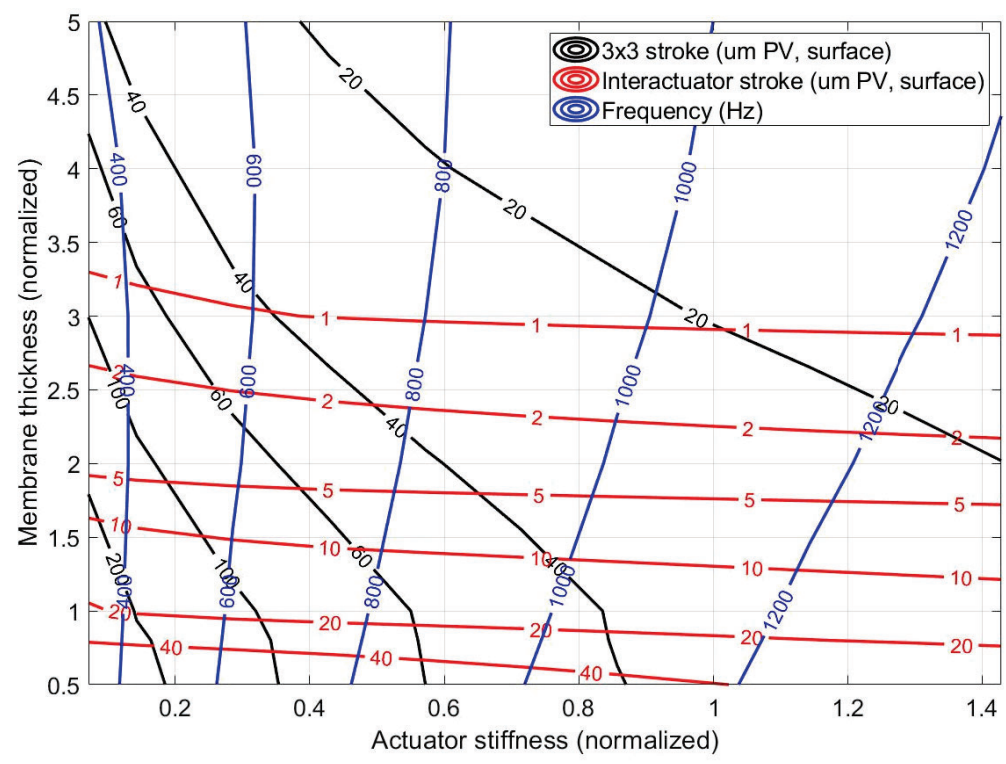

Figure 10: Evolution of DM performance with its characteristics

\section{CONCLUSION AND PERSPECTIVES}

In this paper, we have shown that the performance of ALPAO DMs are compatible with the foreseen requirements for space application.

As an example, we considered a DM with 3228 actuators, organized on a 64x64 regular grid, and an optical pupil of 93 $\mathrm{mm}$ diameter. Without considering the cables and the electronics, the dimensions of such a component is be about 180 $\mathrm{mm} \times 180 \mathrm{~mm} \times 140 \mathrm{~mm}$, and its mass about $5 \mathrm{~kg}$. The power budget will depend on the electronics design, the correction case and the DM's characteristics. To give an order of magnitude, we consider our current standard electronics design and the correction of the surface error presented in Figure 5 (plus the correction of the DM shape at rest). In this case, the power consumed by the $\mathrm{DM}$ is $\sim 1 \mathrm{~W}$. The power dissipated by the electronics will greatly depend on its design, it will need to be adapted according to the constraints.

Currently, our DMs are at TRL4, but work towards space qualification is on-going. For instance, vacuum and cryogenics compatibilities of some components of the system was already demonstrated. Moreover, vibration testing was performed on a DM97-15 and it survived 50G without any impacts on its performance. Figure 11 shows the DM on its test set-up and the measured best flat before and after the test. 


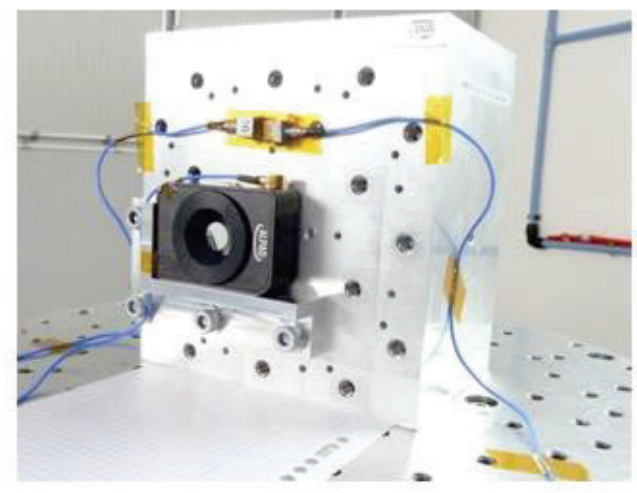

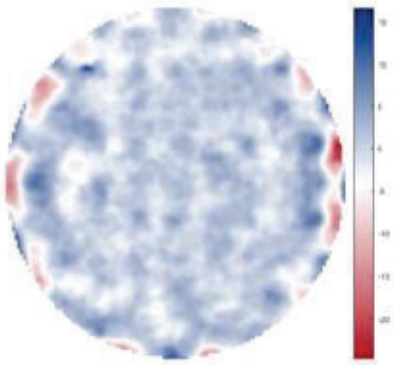

\section{Best flat before vibration test $3.4 \mathrm{~nm}$ RMS}

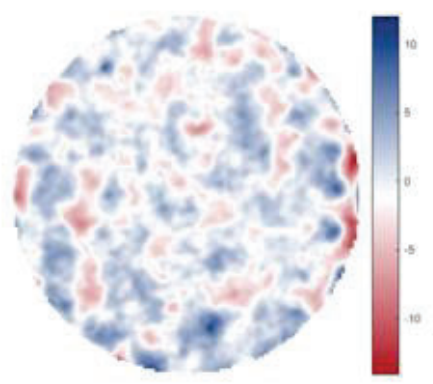

\section{Best flat after vibration test $2.7 \mathrm{~nm}$ RMS}

Figure 11: DM97-15 under vibration test (credit Airbus Defense and Space)

In order to reach a space compatible design, the DM mechanical design needs to be reviewed. The resistance and sensitivity to shock load and vibrations need to be taken into account during the design phase, as well as the thermal and heat management. Another important aspect of this redesign is the weight and size minimization. The different materials (including the mirror coating) that we are classically used might need to be changed depending on the environmental constraints. The stability of the system is also an important point to consider.

For the electronics, space compatible components would need to be chosen for the design, which will have some impact on the performance and the interfaces. Moreover, single point failures need to be investigated in order to propose a reliable design. The reduction of the drive electronics size, the cables weight and number of connections is essential for large number of actuators DMs. For this purpose, an embedded electronics solution is under development. It will have a $80 \%$ efficiency and provide a 18 bits resolution. In such a concept, the drive electronics is directly integrated at the back of the DM (see Figure 12).

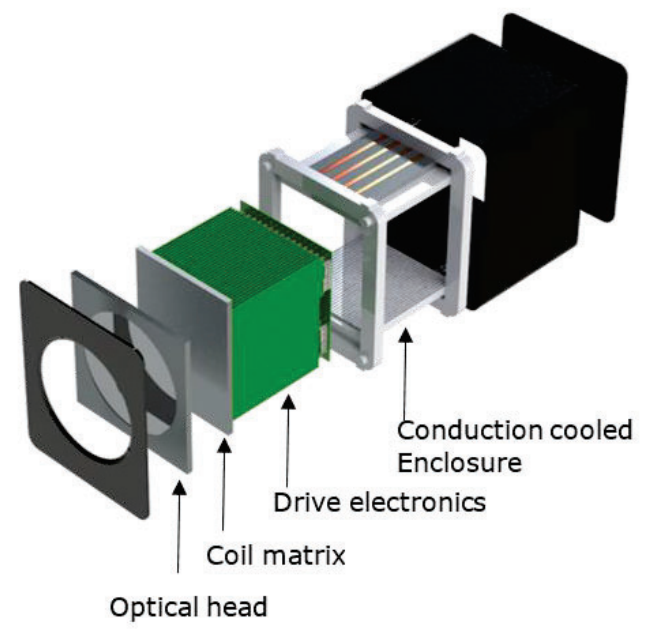

Figure 12: Concept of large DM with embedded electronics 


\section{ACKNOWLEDGMENT}

The author would like to thank Airbus Defense and Space for their collaboration that led to some simulation and experimental results presented in this paper.

\section{REFERENCES}

[1] J. W. Hardy. Adaptive Optics for Astronomical Telescopes. Oxford University Press, July 1998.

[2] M. Postman, T. Brown, K. Sembach, M. Giavalisco, W. Traub, et al. "Advanced Technology Large-Aperture Space Telescope: science drivers and technology developments”. Optical Engineering, 51, January 2012.

[3] M. Laslandes, E. Hugot, M. Ferrari, C. Hourtoule, C. Lopez, F. Chazallet. "Mirror actively deformed and regulated for applications in space: design and performance". Optical Engineering, 52, April 2013.

[4] A. Glindemann, S. Hippler, T. Berkefeld, et al. "Adaptive Optics on Large Telescopes”. Experimental Astronomy 10, April 2000.

[5] F. Vidal, J. Raffard, E. Gendron, S. Thijs, V. Lapeyrère, et al. "Tests and characterisations of the ALPAO $64 \times 64$ deformable mirror, the MICADO-MAORY SCAO AIT facility”. Adaptive Optics for Extremely Large Telescopes conference, 6th edition, November 2019. 